\title{
Peripheral T-cell lymphoma activation on nivolumab for oesophageal cancer
}

\author{
Ian S Boon ${ }^{1}$, Jean S Lim², Karen H Williams³, Kathleen E Romain ${ }^{3}$, Tracy PT Au Yong ${ }^{4}$ and Cheng S Boon ${ }^{5 *}$ \\ ${ }^{1}$ Department of Clinical Oncology, Leeds Cancer Centre, St James's Institute of Oncology, Leeds, United Kingdom \\ ${ }^{2}$ School of Medicine, International Medical University, Kuala Lumpur, Malaysia \\ ${ }^{3}$ Department of Cellular Pathology, Worcestershire Royal Hospital, Worcester, United Kingdom \\ ${ }^{4}$ Department of Radiology, Wirral University Teaching Hospital NHS Foundation Trust, Wirral, United Kingdom \\ ${ }^{5}$ Department of Clinical Oncology, The Clatterbridge Cancer Centre, Birkenhead, United Kingdom
}

\section{Introduction}

Immuno-oncology represents a major paradigm shift in the management of cancer as clinicians move away from targeting cancer cell directly towards targeting the immune system via activation of the human host T-cell $[1,2]$. The efficacy of immunotherapy has been shown in many cancer sites [3]. The activation of $\mathrm{T}$ cells has led to immune-related adverse effects (irAEs) [4]. We report a rare clinical observation of activation of peripheral T-cell lymphoma in a patient treated with Nivolumab.

\section{Case presentation}

This seventh-decade gentleman presented with dyspepsia and dysphagia. His past medical history includes Barrett's oesophagus and osteoarthritis. World Health Organisation (WHO) performance status of zero. Esophagogastroduodenoscopy (OGD) revealed oesophageal cancer which biopsy confirmed moderately differentiated adenocarcinoma (Figure 1). PET/CT confirmed a highly avid midoesophageal cancer (Figure 2) and staged as T3N2M0. HER2 was negative. PET/CT scan further revealed avid left supraclavicular node (Figure 3) which was biopsied and confirmed as high-grade peripheral T-cell lymphoma (PTCL), not otherwise specified (NOS).

Given the dual pathology, oncology multidisciplinary team (MDT) recommended combination chemotherapy for oesophageal cancer which contained anthracycline which PTCL are sensitive. He tolerated 5 cycles of EOX (Epirubicin $50 \mathrm{mg} / \mathrm{mm}^{2}$, Oxaliplatin $130 \mathrm{mg} / \mathrm{mm}^{2}$ and Capecitabine $625 \mathrm{mg} / \mathrm{mm}^{2}$ ). Restaging scan shown partial response with residual disease within the oesophagus and left supraclavicular fossa. MDT decision was to proceed for definitive chemo-radiotherapy with 50 Gy in 25 fractions in combination with weekly Carboplatin (AUC 2) and Paclitaxel $\left(50 \mathrm{mg} / \mathrm{mm}^{2}\right)$. On completion of this, he was given consolidation radiotherapy to the left SCF lymphoma $30 \mathrm{~Gy}$ in 10 fractions.

Restaging scan showed progressive disease with new liver metastases and mediastinal lymphadenopathy. Upper GI MDT and radiologists consensus is that this was consistent with progressive, metastatic adenocarcinoma of the gastrooesophagus rather than T-cell lymphoma progression. Haematology MDT agreed that treatment of the adenocarcinoma would be prioritised given the prognosis of metastatic adenocarcinoma oesophageal cancer was worse than T-cell

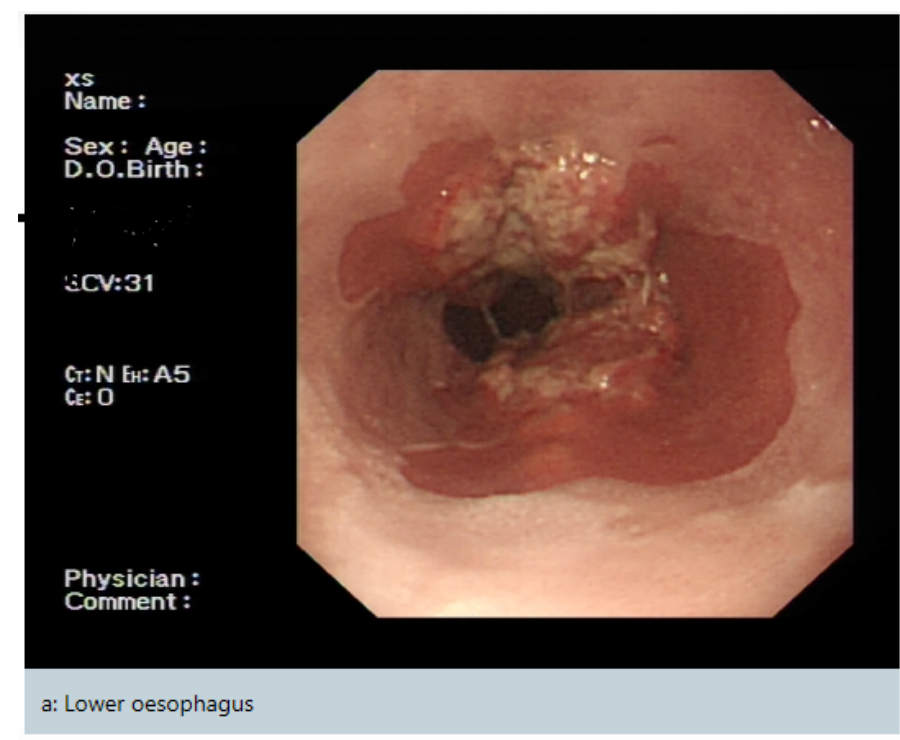

Figure 1. Esophagogastroduodenoscopy (OGD) confirmed a lower oesophageal cancer which in biopsy confirmed moderately differentiated adenocarcinoma

lymphoma. Third line palliative immunotherapy Nivolumab under the early access programme was offered. His WHO performance status at this point was one.

After fourth cycle of Nivolumab, he developed rapidly progressing skin lesions which were thickened and red with the largest over his left anterior chest wall. Dermatology and haematology review suggested this was not classical $\mathrm{T}$ cell lymphoma. In view of clinical progression, we requested a skin biopsy. Biopsy analysis and immunophenotype was confirmed to be peripheral $\mathrm{T}$ cell lymphoma with markers similar to lymphoma at presentation (Figure 4). Patient unfortunately passed away soon after and family declined a post-mortem.

${ }^{*}$ Correspondence to Cheng S Boon, Department of Clinical Oncology, The Clatterbridge Cancer Centre, Birkenhead, United Kingdom, E-mail: cheng. boon@nhs.net

Received: August 05, 2019; Accepted: August 19, 2019; Published: August 22, 2019 


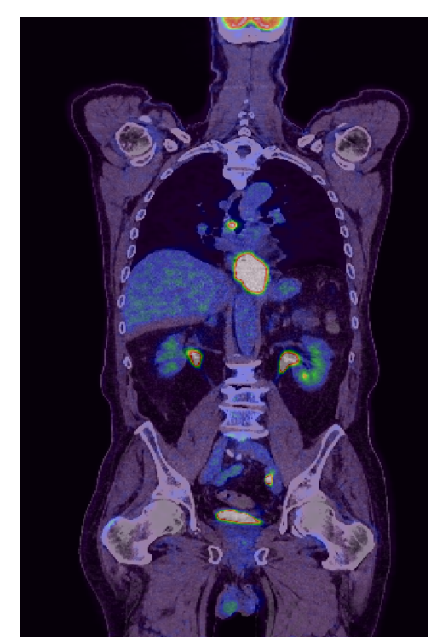

Figure 2. PET/CT scan confirmed a highly avid mid oesophageal lesion giving a staging of T3N2M0



Figure 3. PET/CT scan has revealed a further avid left supraclavicular node which was biopsied and confirmed as high-grade peripheral T-cell lymphoma, not otherwise specified (NOS)

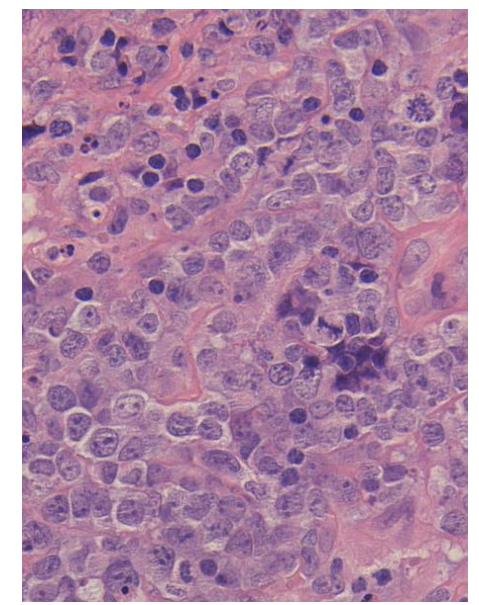

Figure 4. Biopsy analysis with microscopy show a diffuse infiltrate of intermediate sized lymphoid cells in the deep dermis and subcutis. Background small lymphocytes, frequent eosinophils, and neutrophils are seen. There is no conspicuous granulomatous component. Immunohistochemistry revealed CD2 -, CD $3+, \mathrm{CD} 4+, \mathrm{CD} 5$-, CD7 +, CD8 -, PD 1 weak +, CD20 -, CD30 -, CD21 -, CD23 -, PAX5 -, MUM1 -, CD10 -, Ki 67+ (approximately $100 \%$ ), BCL6 weakly +, EBER-. No FDC proliferation is seen with the FDC markers CD21 and CD23. This is consistent with cutaneous involvement by peripheral T-cell lymphoma, NOS

\section{Discussion}

The evidence of palliative immunotherapy with nivolumab in gastric-oesophageal junction cancer comes from a phase 3 trial (ATTRACTION-2) mostly from Asian populations reporting survival benefits [5]. Grade 3-4 toxicities in this trial were $10 \%$ compared against placebo $4 \%$. This was followed by phase 2 CHECKMATE-032 trial in Western cohort again showing superior outcomes [6].

PTCL, not otherwise specific (NOS) is the most common subtype of PTCL (25\%) [7]. It is an aggressive lymphoma with patients often presenting with advanced disease (70\%), high risks of relapse at five years approaching $60-70 \%$ and prognosis are often poor [8,9]. The first line treatment includes anthracycline based combination chemotherapy with cyclophosphamide, doxorubicin, vincristine and prednisone (CHOP). Immunotherapy has also been trialled in haematological cancers such as peripheral T-Cell lymphoma with a Phase $1 \mathrm{~b}$ with nivolumab showing showed a response rate of $40 \%$ but with small numbers of patient [10].

Recent trials have also been developed to identify if radiotherapy can be synergistic when using in combination with radiotherapy with many mechanisms of actions proposed [11]. In our patient, he received radiotherapy prior to starting immunotherapy.

In our patient, he developed rapid cutaneous progression of his PTCL within a short period on commencing Nivolumab while clinically remaining well with no dysphagia to suggest his primary oesophageal cancer progression. As family has declined a post-mortem, we are unable to ascertain cause of death.

Nivolumab acts on PD-1 on T cells which is a check point target to shut down activated T cells. In this case, we hypothesise that Nivolumab by blocking PD-1 can activate normal T cells to attack cancerous cells but theoretically in this patient with T-cell lymphoma could also activate malignant $\mathrm{T}$ cells.

To the best of our knowledge, this is a first case report on peripheral $\mathrm{T}$ cell lymphoma activation while on Nivolumab treatment. We urge all clinicians to be aware of this potentially rare side effect and consider rebiopsy of any sites of suspicious cutaneous or skin manifestations while on immunotherapy to understand the mechanism of this potential interaction.

\section{References}

1. Farkona S, Diamandis EP, Blasutig IM (2016) Cancer immunotherapy: the beginning of the end of cancer? BMC Med 14: 73. [Crossref]

2. Emens LA, Ascierto PA, Darcy PK, Demaria S, Eggermont AMM, et al. (2017) Cancer immunotherapy: Opportunities and challenges in the rapidly evolving clinical landscape. Eur J Cancer 81: 116-129. [Crossref]

3. Eggermont AM, Kroemer G, Zitvogel L (2013) Immunotherapy and the concept of a clinical cure. Eur J Cancer 49: 2965-2967. [Crossref]

4. Postow MA, Sidlow R, Hellmann MD (2018) Immune-Related Adverse Events Associated with Immune Checkpoint Blockade. N Engl J Med 378: 158-168. [Crossref]

5. Kang YK, Boku N, Satoh T, Ryu MH, Chao Y, et al. (2017) Nivolumab in patients with advanced gastric or gastro-oesophageal junction cancer refractory to, or intolerant of, at least two previous chemotherapy regimens (ONO-4538-12, ATTRACTION-2): a randomised, double-blind, placebo-controlled, phase 3 trial. Lancet 390: 2461-2471. [Crossref]

6. Janjigian YY, Bendell J, Calvo E, Kim JW, Ascierto PA, et al. (2018) CheckMate-032 Study: Efficacy and Safety of Nivolumab and Nivolumab Plus Ipilimumab in Patients with Metastatic Esophagogastric Cancer. J Clin Oncol 36: 2836-2844.

7. Broccoli A, Argnani L, Zinzani PL (2017) Peripheral T-cell lymphomas: Focusing on nove agents in relapsed and refractory disease. Cancer Treat Rev 60: 120-129. [Crossref] 
8. Federico M, Bellei M, Marcheselli L, Schwartz M, Manni M, et al. (2018) T cell Project Network. Peripheral T cell lymphoma not otherwise specified (PTCL-NOS). A new prognostic model developed by the International T cell Project Network. Br J Haematol 181: 760-769.

9. Foss FM, Zinzani PL, Vose JM, Gascoyne RD, Rosen ST, et al. (2011) Peripheral T-cell lymphoma. Blood 117: 6756-6767. [Crossref]
10. Lesokhin AM, Ansell SM, Armand P, Scott EC, Halwani A, et al. (2016) Nivolumab in Patients with Relapsed or Refractory Hematologic Malignancy: Preliminary Results of a Phase Ib Study. J Clin Oncol 34: 2698-2704.

11. Martinez-Zubiaurre I, Chalmers AJ, Hellevik T (2018) Radiation-Induced Transformation of Immunoregulatory Networks in the Tumor Stroma. Fron Immunol 9: 1679. [Crossref]

Copyright: (C2019 Boon IS. This is an open-access article distributed under the terms of the Creative Commons Attribution License, which permits unrestricted use, distribution, and reproduction in any medium, provided the original author and source are credited. 